\title{
When teaching is not enough: Exploring educational computer games as a method for improving reading ability
}

\author{
Laura Lee
}

School of Applied Psychology, UCC

\section{The problem}

Imagine for a moment that you are a child once again, sitting in your primary school classroom. 30 other students are sitting with you, all at desks working at a task set by your teacher. You are reasonably content, happy to work independently on the task at hand. Suddenly, your teacher announces that it is time to put your workbooks away; you are moving on to the next lesson of the day. The lesson is English and your teacher has asked that you start the lesson by reading aloud to the class from your textbook. Panic creeps up from the pit of your stomach, your heart races and your palms start to sweat. Why? Because you know that you cannot read as well as the others in your class. In fact, you know that you cannot read as well as most other children your age. For many of your friends, reading is quick, accurate and effortless. For you, it is a slow, inaccurate, tedious endeavour that frequently causes frustration, anxiety, and embarrassment.

I think you will agree that the above scenario is definitely unpleasant. Unfortunately, though, it is one that many children encounter on a regular basis. Indeed, there are countless children in classrooms all over the world for whom reading is a major difficulty. Such difficulty with reading is not only distressing, but also likely to affect performance in a variety of school subjects; if you can't read the text, how can you understand the content? To add to this difficulty, many Irish schools have experienced drastic resource cuts in recent years. The number of children in need of support remains constant yet the resources available for such students and their teachers continues to dwindle.

\section{Fixing the problem}

Psychologists all over the world have been studying reading for a great number of years. Much of this research has focused on figuring out why some children find it easier to read than others. We know a lot about the causes of those differences, both environmental (e.g. family literacy practices) as well as those that lie within the child (e.g. the ability to process the sounds of speech). However, a clear understanding of how this research can 
be translated into feasible, teacher friendly practices is much less apparent. Thus, psychologists and educators are still trying to determine the best methods to support children who struggle with reading.

One belief that has gained increasing attention in recent years is that computers can be used to effectively improve the reading performance of struggling readers. This is because they can provide individual, specialised instruction, are cost effective, and often don't require adult supervision. Furthermore, a lot of children enjoy using computers, so their motivation to work on a particular skill and enjoyment whilst doing so are likely to be increased. There are also benefits for teachers, who experience less repetition and drudgery in their teaching and have access to accurate documentation of student progress.

Quite a lot of research has been undertaken to investigate whether computers can be effectively used to improve the skills needed by children to become proficient readers. However, consensus has not yet been reached as to whether or not computer programs designed to teach children reading related skills are truly effective for improving reading ability. Several researchers have found that these types of computer programs have a small to moderate positive effect on reading ability but others have found no such effect. As part of my $\mathrm{PhD}$, I am investigating whether or not the educational computer game "GraphoGame-Fluent" is an effective form of remediation for struggling readers.

\section{GraphoGame-Fluent}

The educational computer programme "GraphoGame" was originally developed in Finland as a result of The Jyväskylä Longitudinal Study of Dyslexia Project. The original GraphoGame software was designed to assist children at the very start of their formal education. Its purpose was to support children learning correspondences between letters and sounds. The training was computerised and available online free of charge in the hope that those in need could be more easily reached as it did not require the presence of a supervising adult. It was also hoped that the use of computerised training would create a "more play, less work" environment, encouraging sustained attention and involvement from the child.

GraphoGame-Fluent is an expansion of the original GraphoGame software and was developed by a team of researchers from Finland, Ireland, and Austria. The aim of GraphoGameFluent is to support older children from the 3rd to 6th class who experience persistent reading fluency difficulties in English.

The game is based on the idea that in order to become a truly proficient reader, children need repeated, consistent reading practice. Many children who struggle with reading have problems with accurate and automatic (fast and effortless) word recognition. GraphoGame-Fluent works to alleviate these difficulties by repeatedly exposing the play- 
ers to high frequency English words which they are likely to frequently encounter in text. Also, the game includes an adaptive algorithm which adjusts the speed of item presentation to the player's response accuracy. This means that the players are always challenged to play at the edge of their ability. Furthermore, the game aims to improve the connection between spoken and written language, another difficulty faced by many poor readers.

GraphoGame-Fluent is a role playing game that consists of a number of different levels (a jungle, castle etc). The player assumes a character and progresses through each level by completing training tasks involving high frequency English words and sentences. The tasks include:

Spoken to written word matching (the player hears a word in their earphones and matches it to the correct word on screen);

Written to written word matching (the player sees two words in quick succession and must decide if they are the same or different);

Sentence reading (the player decides if a sentence they see on screen is true or false);

Word organisation (the player sees words on screen and must decide which ones belong to a certain category).

Players receive feedback in the form of a result chart after each training task. The players also earn money as they complete training tasks. They can use this money in the many shops contained within the game; clothes, jewellery, pets, and motorcycles are all available for purchase!

\section{The study}

In order to find out whether GraphoGame-Fluent could actually improve reading ability, a group of 43 primary school students were asked to play the game. All of the students had been nominated by their teachers as poor readers. They were aged between 8 and 12 years old and were in the 3rd to 6 th classes. The group of nominated students was then divided into two. One group played GraphoGame-Fluent from February-March 2013 while the other played from April-May 2013.

Participants played GraphoGame-Fluent either at home, at school or both. Playing was encouraged for 25 minutes a day (Monday to Friday) for 7 weeks. An online assessment built into the game provided an evaluation of children's reading skills at 3 points throughout the intervention period (January (T1), April (T2) and June (T3)).

A group of 43 randomly selected average readers from the same classes were also recruited as a comparison group. These students did not play GraphoGame-Fluent but did complete 
the 3 assessments. This allowed me to compare the performance of the poor readers playing GraphoGame-Fluent to the performance of their classmates.

\section{Did it work?}

The online assessment that took place at three time points allowed me to measure children's ability on three different reading measures and two control measures. Each player's ability was assessed in terms of time taken to complete the tasks and performance accuracy.

The first assessment allowed me to collect what is called a "baseline" measure of the participant's performance before either group started playing GraphoGame-Fluent. At this time, no significant differences were observed between the two groups of poor readers in terms of response time or accuracy.

The second assessment took place just as the early playing group had stopped playing and the late playing group had yet to start. At this time, results showed that the early playing group were significantly faster than the late playing group on two of the reading measures and one of the control measures. It seems that GraphoGame-Fluent was effective for reducing the reaction time of the players.

At the time of the third and final assessment, when the late playing group had just finished playing, no significant differences were found between either of the poor reader groups in terms of response time or accuracy on any of the measures. At T3, both poor reader groups performed at a faster rate than they had done at T2. However, the accuracy of both groups was worse at T3 than it had been at T2. Although reaction time seemed to be decreasing, so too was accuracy! This may have been due to the fact that participants were completing the same assessment for a third time; eagerness to finish the assessment may have caused a speedy but inaccurate response. At T3, both poor reader groups were still not as accurate as the comparison group of average readers.

Unfortunately, I was disappointed to find out that participants did not play the game as much as intended. Overall playing time for the early group was better (5.3 hours) than for the late playing group (4.3 hours) but neither reached targeted levels (14.5 hours). Children participating in the study played largely unsupervised at home. It appears that playing which involves adult supervision in a group setting where player interaction is possible provides the most promise for sustained playing. 


\section{What did the players make of the games?}

In order to find out what the participants thought of GraphoGame-Fluent, I carried out three focus groups. Within each focus group, I spoke with between four and six students about their experience of the game. The participants had several suggestions for how the game could be improved in the future. Some of these suggestions included:

\section{Increased opportunity for interaction:}

"Let's say your friend was on it and... you could add them as a friend and then... you could play with them"

\section{Diversity within the game:}

"Do maths tasks, English and everything"

\section{Increased opportunity for reward:}

"I wanted more money!"

\section{Increased creative input:}

"I think we should make our own characters"

\section{"Real World" research}

Carrying out research is often a complex process with each type of research posing different challenges. Because this study involved participants playing Graphogame-Fluent every day in school or at home, it faced some challenges associated with this type of context. For me, the following questions needed to be considered carefully:

$\square$ Will the player's parents be able to download the game easily?

$\square$ Do the participants have access to suitable facilities at home and/or school to reliably and consistently play the game?

Are adults available to supervise the players if necessary?

Do the participants have the time and motivation to play the game? 
For some participants, the answers to the above questions were sometimes "No!" For example, there were several children who had computers at home which subsequently broke, denying them access to the game. For others, the amount of extra-curricular activities they were engaged in sometimes meant their schedule was too full to play! Furthermore, despite a lot of encouragement on my part, some participants were not very motivated to play. Thus, the research process needed to be flexible and adaptive, ensuring the participants were granted as much exposure to the game as possible. For example, sessions were arranged within the school where participants would play the game under my supervision. This was to ensure that participants had at least some exposure to the game. It is thought that player motivation can perhaps be improved by altering the game in the ways suggested by the players e.g. facilitating interaction between players.

\section{What have we learned?}

When assessed at T2, the group who had been playing GraphoGame-Fluent were significantly faster on three tasks (two reading tasks and one control task) than the group who had yet to start playing. This suggests that GraphoGame-Fluent had a positive effect on player's ability to respond to tasks more quickly. At T3, the poor reader groups did not differ significantly. Results indicate that whilst player speed had increased between T2 and T3, accuracy had decreased during that time. It still remains to be seen how effective GraphoGame-Fluent can be when played consistently over a prolonged period of time. Future research in this area will be informed by the valuable suggestions provided by the players and also a consideration of the practical constraints involved when offering educational intervention online.

I would like to extend my thanks to my supervisor Dr. Marcin Szczerbinski, the GraphoGameFluent team, and the participants, parents, and staff who made my research possible. 